\title{
Identifying Extreme PAPR in Coherent Optical Communications
}

\author{
S.A. Derevyanko(1), A. Redyuk ${ }^{(2,3)}$, S. Vergeles ${ }^{(4,5)}$, S. Turitsyn ${ }^{(2,6)}$ \\ (1) Department of Electrical and Computer Engineering, Ben Gurion University of the Negev, Beer \\ Sheva 84105, Israel, stasd@bgu.ac.il \\ (2) Novosibirsk State University, Novosibirsk 630090, Russia. \\ (3) Institute of Computational Technologies SB RAS, Novosibirsk 630090, Russia. \\ (4) Landau Institute for Theoretical Physics RAS, Moscow 119334, Russia. \\ (5) Moscow Institute of Physics and Technology, Dolgoprudnyj 141700, Russia. \\ (6) Aston Institute of Photonic Technologies, Aston University, Birmingham B4 7ET, UK.
}

\begin{abstract}
We apply well established concepts of adaptive wave front shaping used in imaging through turbid medium to detect detrimental phase modulated sequences in multi-carrier optical communications that can cause extreme power fluctuations due to dispersion enhanced interference of information symbols.
\end{abstract}

\section{Introduction}

Optical signal carrying information is characterised by statistical distributions that are changing with distance, as signal propagates along the optical fiber. Signal properties are affected by fiber communication channel loss/gain, dispersion and nonlinear effects. Statistical fluctuations in telecommunication data streams occur both due to information content of optical signal and overlapping of many symbols that occurs due to dispersive broadening of carriers. Exploitation of optical phase and multilevel data coding in coherent communications systems leads to very irregular waveforms with substantial statistical variance of power (high peak-to-average-power ratio, PAPR) ${ }^{1}$ that can bring about completely new challenges compared to previous system designs. Extreme value fluctuations of signal power being rare events still can have major detrimental consequences.

Here we focus on identifying dangerous phasecoded sequences that lead to those rare events. Rather than dealing with the probabilistic approach to PAPR statistics we concentrate on calculating analytically the initial phase patterns that generate extreme peak power outage at a given point of the fiber. To achieve that goal we have transferred here some well-known methods of diffraction optics to the field of optical communications. Knowledge of such patterns leading to large power fluctuations at the nondesirable points (nonlinear elements, amplifiers, and so on) can be used in signal pre-processing or coding ${ }^{2}$, effectively removing the most dangerous combinations.

\section{Theoretical model}

We have used standard nonlinear Schrödinger equation model as the basic path-averaged model: $\frac{\partial E}{\partial z}+i \frac{\beta_{2}}{2} \frac{\partial^{2} E}{\partial t^{2}}-i \gamma|E|^{2} E=N(z, t)$,

where the standard telecom parameters are assumed for the second order dispersion and nonlinearity coefficients: $\beta_{2}=-20.4 \mathrm{ps}^{2} / \mathrm{km}$, $\gamma=1.23(\mathrm{~W} \mathrm{~km})^{-1}$. Here we have also assumed either path-averaged description or the ideal distributed Raman amplification with the amplifier spontaneous emission term $N$.

We considered initial signals using the orthogonal frequency division multiplexing format (OFDM) ${ }^{1}$ :

$$
E(z=0, t)=\sum_{\alpha=1}^{N_{s c}} \sum_{n=1}^{N} c_{n \alpha} s\left(t-n T_{s}\right) e^{2 \pi i f_{\alpha^{t}}}
$$

where $c_{n \alpha}=\left|c_{n \alpha}\right| \exp \left(i \theta_{n \alpha}\right)$ are the set of the modulation symbols, $1 / T_{s}$ is a symbol rate, $s(t)$ is the rectangular shape of the envelope carrier and the subcarrier spacing is chosen to be $f_{\alpha+1}-f_{\alpha}=1 / T_{s}$ to ensure orthogonality. No cyclic prefix is assumed here for simplicity, but results can be easily generalised to include it.

We consider here as an example of the general theory M-PSK modulation and ring constellation format, where $c_{n \alpha}$ are uniformly distributed on a series of given circles of fixed radii.

\section{Theoretical results}

Traditionally PAPR studies concentrate on the statistical analysis of the sum of different subcarriers inside a single slot $n$. However, fiber channel has important difference from its wireless counterpart which makes PAPR analysis different. First, fiber channels are subjected to distributed Kerr nonlinearity. Second, during the signal propagation due to fiber dispersion different slots overlap which 

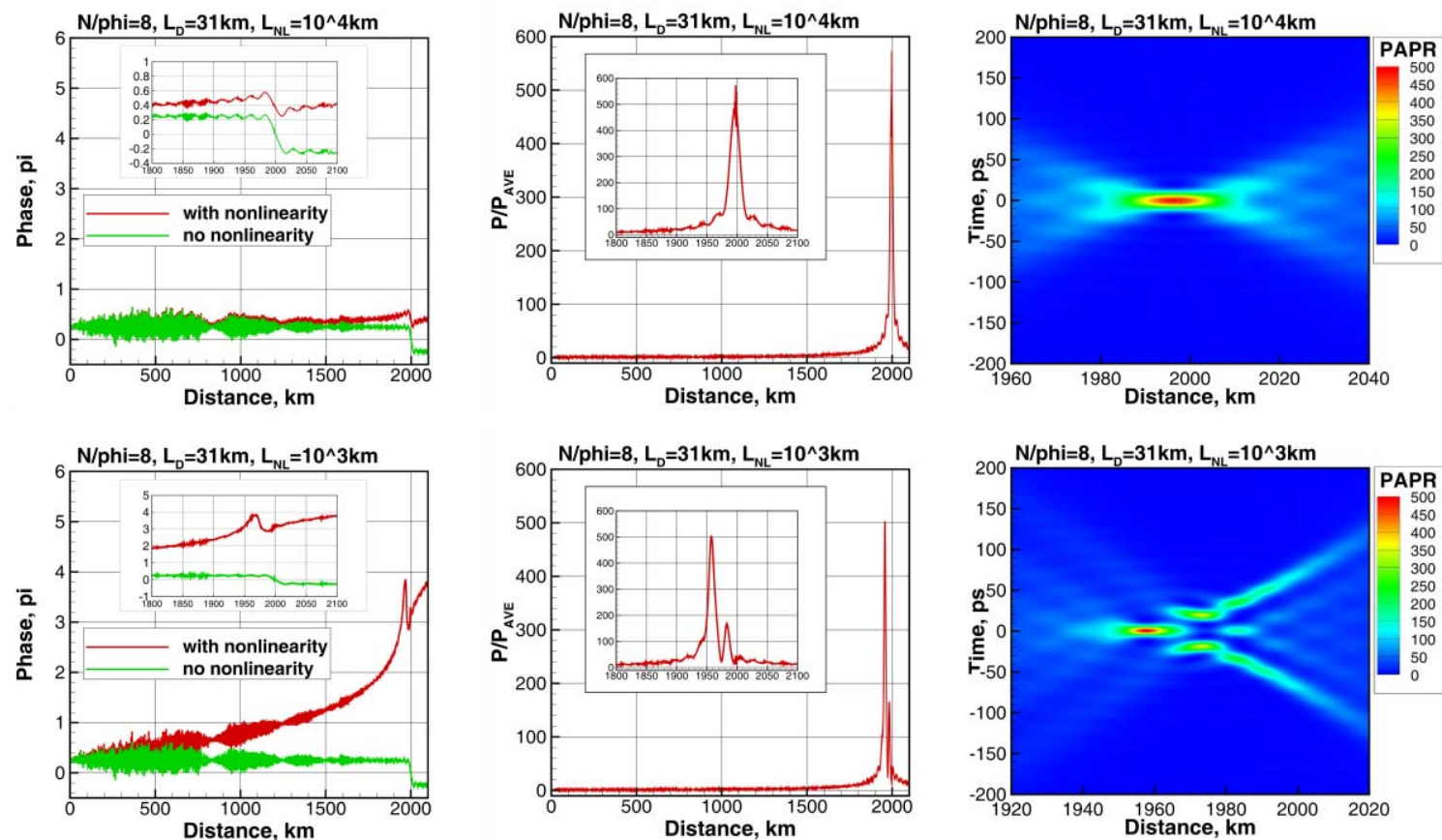

Fig. 1: Dependence of signal phase (left column) and power (center column) on distance for target point $t *=0$, spatio-temporal dynamics of the RW (right column) single subcarrier average input power $-11 \mathrm{dBm}$ (top) and $-1 \mathrm{dBm}$ (bottom).

provides additional degrees of freedom leading to strong power fluctuations. For single carrier transmission this effect was studied in $\mathrm{Ref}^{3}$. A typical extreme event has an appearance of a sharp peak in the $(z, t)$ plane appearing "out of nowhere" and disappearing "without a trace" - a typical example is given by the right column in Fig. 1. This makes an interesting analogy with an actively discussed topic of "freak" (or "rogue") waves in optical fiber systems ${ }^{4}$ that in turn were studied in analogy to the giant waves in the ocean. Of course, such giant power fluctuations are undesirable, especially when they occur at the points of amplification. The simulated power fluctuations like the ones given in the right column of Fig. 1 occur for a non-trivial, specific and rare combinations of the initial phases in the OFDM signal (2). To determine (aiming to prevent them) such dangerous combinations, it would require a multidimensional optimization of the field power $P=|E|^{2}$ at a given space-time point $\left(L, t_{*}\right)$ over the phases of the subcarriers at each symbol in (2). Generally it is extremely computationally expensive. However when the nonlinearity can be neglected the linear input-output relation make analytical solution possible. In this case the target instantaneous power can be written as a quadratic function of the individual symbols:

$$
P\left(L, t_{*}\right)=\left|\sum_{n, \alpha} T_{n \alpha}\left(L, t_{*}\right) e^{i \theta_{n \alpha}}\right|^{2},
$$

where $T_{n \alpha}$ have the meaning of transmission coefficients. This provides a cross-disciplinary connection to the problem of adaptive wave-front shaping in diffraction optics where similar optimization of field transferred through disordered media is obtained by optimizing the phases of the pixels of spatial light modulator ${ }^{5}$. Regardless of the physical system the "dangerous" phases leading to coherent addition of the contribution from different slots and subcarriers are obtained by phase conjugation of the transmission coefficient: $\theta_{n \alpha}=-\operatorname{Arg}\left(T_{n \alpha}\right)$. In the case of dispersion-affected linear propagation of pulses the transmission coefficients are available analytically which provides us with full analytical description of the problem. Omitting the details we present the main results for the continuous initial phases and the resulting PAPR for the generated power outage:

$$
\begin{gathered}
\theta_{n \alpha}=-\operatorname{Arg}\left[\tilde{s}_{n \alpha}\left(t_{*}\right)\right]+\frac{n T_{s}\left(n T_{s}-2 t_{*}\right)}{2\left|\beta_{2} L\right|} \\
P A P R=\frac{\left(\sum_{\alpha=1}^{N_{s c}} \sum_{n=1}^{N}\left|T_{n \alpha}\right|\right)^{2}}{\sum_{\alpha=1}^{N_{s c}} \sum_{n=1}^{N}\left|T_{n \alpha}\right|^{2}} \equiv \varphi \Omega\left[N_{s c}, N / \varphi\right]
\end{gathered}
$$

where $\tilde{s}_{n \alpha}$ is the Fourier transform of the carrier shape evaluated at the argument $f_{\alpha}+(t-n T s) /\left(2 \pi \beta_{2} L\right)$ and we have introduced an important symbol overlap parameter $\varphi=\left|\beta_{2} L\right| / T_{s}^{2}$ and for uncompensated transmission $\varphi>>1$. The resulting PAPR is proportional to this overlap parameter but also contains an additional formfactor $\Omega$ that takes into account the finite number 
of symbols and subcarriers. We have derived a closed form analytical expression for this factor in most cases of interest which are not presented here due to space considerations. It is also possible to obtain a closed from expression for the spatio-temporal shape of the resulting "freak wave". Eq. (4) provides analytical solution for the case of continuous field modulation. For the MPSK transmission one simply selects the closest constellation point.

When the power grows to higher levels the nonlinear effects are triggered making fluctuations irreversible. To examine the impact of nonlinearity on such fluctuations we have undertook a series of additional simulations with progressively increasing signal power (in other words, level of nonlinearity) - Fig.1. In all the cases the initial phases were selected according to Eq. (4) for target point $L=2000 \mathrm{~km}, t_{*}=0$.

Fig. 1 shows dependence of signal phase on distance at the target time point $t=t_{*}$ (left column), dependence of the power at the same point on the distance (center column) and spatiotemporal dynamics of the "freak wave" (right column). One can see that at moderate powers the linear result (4), (5) successfully holds and predicts a correct power outage at a given spatiotemporal location. At higher signal power an additional phase shift occurs as shown by read curves in the left column. This additional nonlinear phase shift leads to shift in spatial position of the flucuation from expected distance $L=2000$. Thus, we conclude that the fiber nonlinearity makes high power fluctuations irreversible and leads to substantial signal distortion.

\section{Impact on system performance}

To estimate the impact of such extreme events on the system performance we performed a set of numerical simulations. We considered a $25 \times 100 \mathrm{~km}$ transmission link with an ideal distributed Raman amplification. As an input we consider QPSK-OFDM signal with 16 subcarriers, OFDM base $T_{s}=100 \mathrm{ps}$ and the two types of initial bit stream: random binary sequence and predefined binary sequence. The former is determined by pseudorandom process, whereas the second one is determined by (2) and (4) and leads to high power fluctuation at preselected intermediate distance of $2000 \mathrm{~km}$. At the receiver the chromatic dispersion was fully compensated and for phase estimation, an algorithm based on the 4th-power Viterbi-Viterbi method was used. To evaluate the system performance we use the $\mathrm{Q}$-factor evaluated via error vector magnitude. Fig 2 shows the system performance for both random and predefined bit streams. It can be seen that the optimal power difference reaches the value of $5 \mathrm{~dB}$. Furthermore, occurrence and dissipation of high

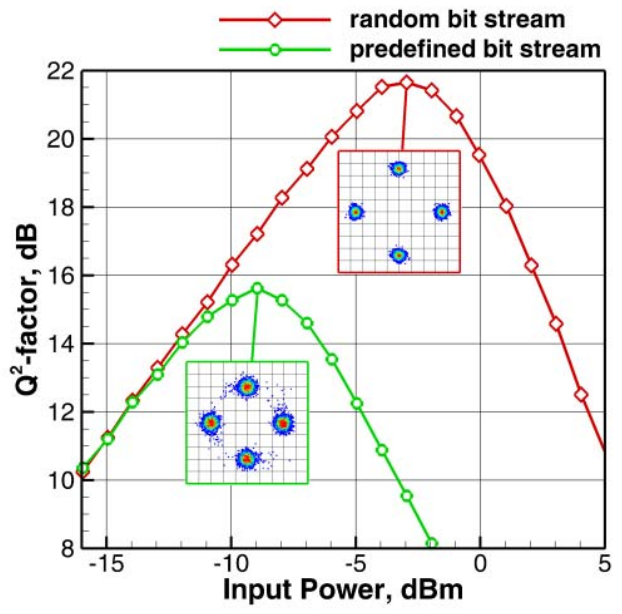

Fig. 2: System performance as a function of the signal input power for random binary sequence (red) and predefined binary sequence (green).

power rogue wave during signal transmission results in $6 \mathrm{~dB} \mathrm{Q}^{2}$-factor penalty due to added nonlinear signal distortion.

\section{Conclusions}

In this work we have identified a straightforward procedure for finding "dangerous" sequences of phase-coded symbols that lead to occurrence of localized high power fluctuations at certain propagation distances. Knowledge of such extreme high-PAPR fluctuations is important for their prevention.

\section{Acknowledgements}

This work was supported by the Russian Science Foundation (Grant No. 17-72-30006).

\section{References}

[1] W. Shieh and I. Djordjevic, OFDM for Optical Communications, Elesevier (2010).

[2] A. Shafarenko et al., "Information-theory analysis of skewed coding for suppression of pattern-dependent errors in digital communications," IEEE T. Commun., Vol. 55, no. 2, p. 237 (2007).

[3] S. Vergeles and S. Turitsyn, "Optical rogue waves in telecommunication data streams," Phys. Rev. A, Vol 83, no. 6, Art. No. 061801 (2011).

[4] J. M. Dudley et al., "Instabilities, breathers and rogue waves in optics," Nat. Photonics., Vol. 8, p. 755 (2014).

[5] I. M. Vellekoop and A. P. Mosk., "Focusing coherent light through opaque strongly scattering media," Opt. Lett., Vol. 32, no. 16 p. 2309 (2007). 